EUROPEAN ORGANIZATION FOR NUCLEAR RESEARCH

European Laboratory for Particle Physics

\title{
MODELLING OF HELIUM-MEDIATED QUENCH PROPAGATION IN THE LHC PROTOTYPE TEST STRING-1
}

\author{
M. Chorowski ${ }^{1}$, P. Grzegory ${ }^{2}$, L. Serio ${ }^{2}$ and R. van Weelderen ${ }^{2}$
}

\begin{abstract}
The Large Hadron Collider (LHC) prototype test string-1, hereafter referred to as the string, is composed of three ten-meter long prototype dipole magnets and one six-meter long prototype quadrupole magnet. The magnets are immersed in a pressurized static bath of superfluid helium that is maintained at a pressure of about 1 bar and at a temperature of about $1.9 \mathrm{~K}$. This helium bath constitutes one single hydraulic unit, extending along the $42.5 \mathrm{~m}$ of the string length. We have measured the triggering of quenches of the string magnets due to the quenching of a single dipole magnet located at the string's extremity; i.e. "quench propagation". Previously reported measurements enabled to establish that in this configuration the quench propagation is mediated by the helium and not by the inter-magnet busbar connections [1], [2]. We present a model of helium mediated quench propagation based on the qualitative conclusions of these two previous papers, and on additional information gained from a dedicated series of quench propagation measurements that were not previously reported. We will discuss the specific mechanisms and their main parameters involved at different time scales of the propagation process, and apply the model to make quantitative predictions.
\end{abstract}

1 University of Technology, Wybrzeze Wyspianskiego 27, 50-370 Wroclaw, Poland

2 CERN, LHC Division, Geneva, Switzerland

Presented at CHATS-Y2K: Workshop on Computation of tHermo-hydrAulic Transients in Superconductors September 6-9, 2000, Frascati, Italy

\footnotetext{
Administrative Secretariat

LHC Division

CERN

CH - 1211 Geneva 23

Switzerland

Geneva, 23 March 2001
} 


\title{
Modelling of helium-mediated quench propa- gation in the LHC prototype test string-1
}

\author{
M. Chorowski †, P. Grzegory, L. Serio and R. van Weelderen* \\ CERN, LHC Division, CH-1211 Geneva 23, Switzerland \\ †University of Technology, Wybrzeze Wyspianskiego 27, 50-370 \\ Wroclaw, Poland
}

The Large Hadron Collider (LHC) prototype test string-1, hereafter referred to as the string, is composed of three ten-meter long prototype dipole magnets and one sixmeter long prototype quadrupole magnet. The magnets are immersed in a pressurized static bath of superfluid helium that is maintained at a pressure of about 1 bar and at a temperature of about $1.9 \mathrm{~K}$. This helium bath constitutes one single hydraulic unit, extending along the $42.5 \mathrm{~m}$ of the string length. We have measured the triggering of quenches of the string magnets due to the quenching of a single dipole magnet located at the string's extremity; i.e. "quench propagation". Previously reported measurements enabled to establish that in this configuration the quench propagation is mediated by the helium and not by the inter-magnet busbar connections [1], [2]. We present a model of helium mediated quench propagation based on the qualitative conclusions of these two previous papers, and on additional information gained from a dedicated series of quench propagation measurements that were not previously reported. We will discuss the specific mechanisms and their main parameters involved at different time scales of the propagation process, and apply the model to make quantitative predictions.

Keywords: quench $(C)$; propagation velocity $(C)$; superconducting magnets $(E)$; superfluid helium (B); supercritical helium (B)

\footnotetext{
*To whom correspondence should be addressed.
} 


\section{Notation}

$A_{\mathrm{e}}(a)$ : free endcap cross-sectional area $0.0363 \mathrm{~m}^{2}$

$A_{\mathrm{e}}(b)$ : free endcap cross-sectional area $0.042 \mathrm{~m}^{2}$

$A_{\mathrm{i}} \quad$ : free interconnection cross-sectional area $0.0029 \mathrm{~m}^{2}$

$A_{\mathrm{y}} \quad:$ free yoke cross-sectional area $0.021 \mathrm{~m}^{2}$

$I \quad$ : electrical current

$L_{\mathrm{c}} \quad$ : distance yoke-end to coil peak field location 0.14-0.15 m

$L_{\mathrm{e}}(a)$ : endcap length $0.275 \mathrm{~m}$

$L_{\mathrm{e}}(b)$ : endcap length $0.190 \mathrm{~m}$

$L_{\mathrm{i}} \quad$ : interconnection length $0.44 \mathrm{~m}$

$L_{\mathrm{y}} \quad$ : yoke length $10 \mathrm{~m}$

$L_{\text {string }}$ : string length $42.5 \mathrm{~m}$

$L_{\text {dipole }}$ : dipole cold mass length $10.9 \mathrm{~m}$

$L_{\text {rst }} \quad: \quad L_{\text {string }}-L_{\text {dipole }}$

$U \quad$ : internal energy

$V \quad$ : volume

$h \quad: \quad$ specific enthalpy

$m$ : mass

$p \quad:$ pressure

$p_{\text {set }} \quad: \quad$ SRV set pressure

$v \quad:$ velocity

$\rho \quad:$ mass density

\section{Introduction}

CERN is building the Large Hadron Collider (LHC), a proton and ion collider with center-ofmass energy in the TeV-per-constituent range. This machine, to be installed close to Geneva (Switzerland) in the $26.7 \mathrm{~km}$ circumference tunnel of the previous LEP collider, will accelerate and bring into collision intense beams of protons and ions at higher energies than ever achieved before. The LHC will make intensive use of high-field, twin-aperture superconducting magnets, operating in static baths of pressurized helium II at $1.9 \mathrm{~K}$ and at about $0.1 \mathrm{MPa}$. The regular LHC lattice will consist of $53.5 \mathrm{~m}$ long half-cells comprising a quadrupole magnet and three dipole magnets. Two half-cells constitute a $107 \mathrm{~m}$-long single hydraulic unit. The LHC cryogenic scheme and the concept of magnet cooling is described in detail in [3].

The protection of the LHC superconducting main dipole and quadrupole magnets in case of a quench is based on quench heaters and cold by-pass diodes [4], [5]. Once a quench is detected, the quench heaters are fired to warm up a large fraction of the outer coils, provoking a large electric resistance growth. The current commutates from the quenching magnet over to the cold by-pass diodes connected in parallel. The magnets that do not quench are de-excited by switching off the power converter and opening switches, with resistances in parallel. The time constant for the de-excitation is about $100 \mathrm{~s}$ for the dipole magnet circuit and about $45 \mathrm{~s}$ for each of the quadrupole magnet circuits. 


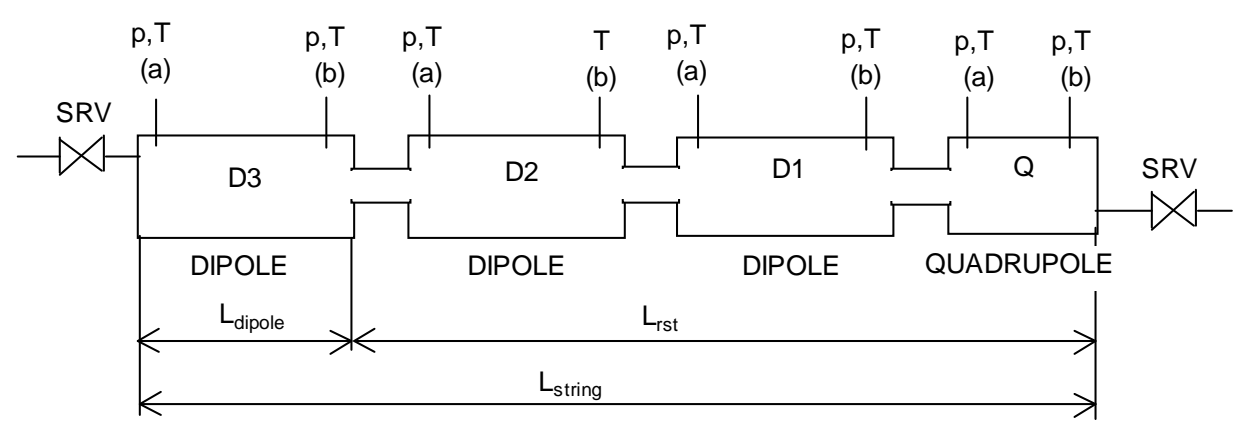

Figure 1: Schematic of the configuration of the LHC prototype test string-1.

The evolution of the helium in the cold mass of the quenched magnet has been observed and then described with a mathematical model based on energy conservation [6]. The thermohydraulics of a magnet resistive transition is governed by two main processes: initially a fast adiabatic compression of the bulk of helium by the rapidly expanding portion of helium in the vicinity of the coil. Within some milliseconds after the resistive transition the pressure and the temperature in the cold mass homogenize and the helium can be treated as a lumped energy source for the adjacent cold masses. During the time required for the discharge, other magnets might quench due to thermo-hydraulic propagation in the helium bath or heat conduction via the bus bars. The number of quenching magnets depends on the mechanism for the propagation and should be limited to the minimum. In the LHC, the bus bar length in between two magnets will be about $15 \mathrm{~m}$. It was concluded from previously reported measurements [1], [2] that, because of this long bus bar length, quench propagation will be mediated by the helium only. The aim of the present study is to establish a model for helium-mediated quench propagation and to provide the main process parameters. For this we base ourselves on the qualitative conclusions from the two previous papers [1], [2], and on additional information gained from a dedicated series of quench propagation measurements that were not previously reported.

\section{The LHC prototype test string-1}

\section{Layout}

Within the R\&D program for the Large Hadron Collider, a string of three 10-m long dipole magnets (D1, D2, D3) and one 3-m long quadrupole magnet (Q) has been assembled and tested at CERN [7]. The helium bath in which these magnets are immersed constitutes one single hydraulic unit, extending along $42.5 \mathrm{~m}$ of the string length. Its total helium volume is $0.850 \mathrm{~m}^{3}$ (Figure 1).

The configuration of cold by-pass diodes protecting the string magnets enabled us to either provoke a resistive transition of $\mathrm{Q}$ and to observe quench propagation to D1, or to quench D3 and to observe quench propagation to D2 [8]. Pressure relief can take place at either end of the string through spring-loaded safety relief valves (SRV's) and at adjustable pressure setting levels. 


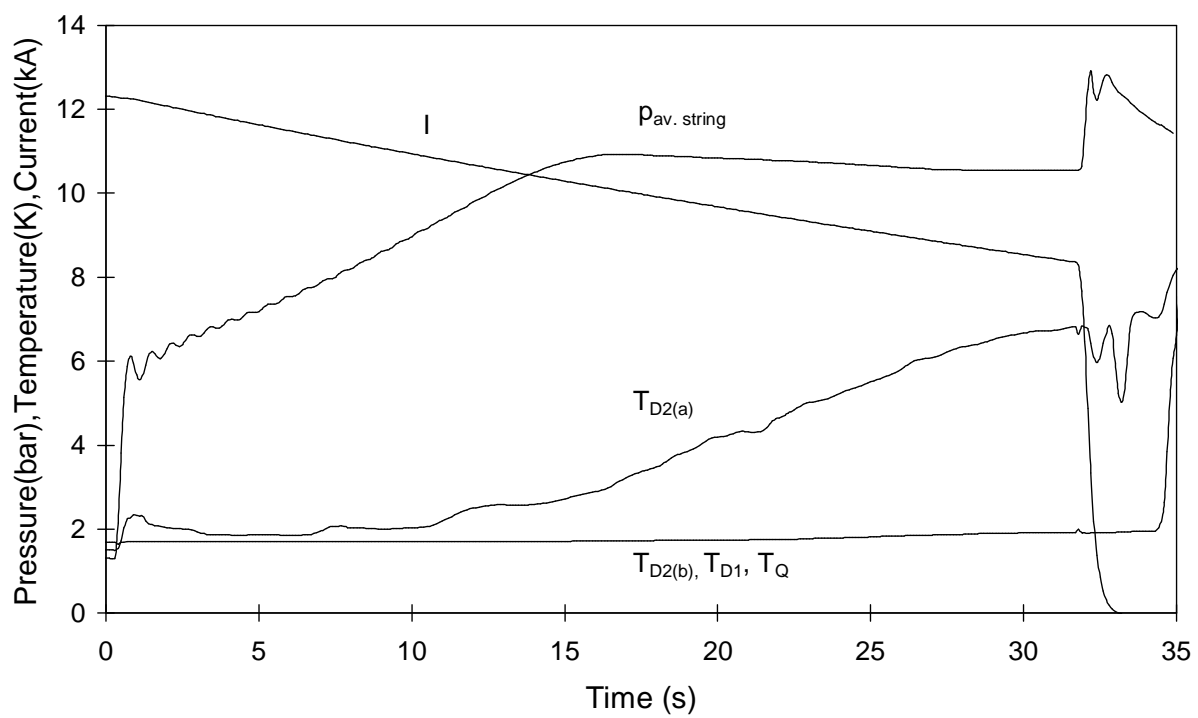

Figure 2: Quench propagation from D3 to D2; $p_{\text {set }}=11$ bar.

\section{Instrumentation}

Pressure and temperature probes (Allen-Bradley carbon resistors, $100 \Omega$ platinum resistors, and piezo-resistive cold pressure sensors) in the end volumes of each magnet are glued to the end plates at the extremities of the coil assemblies. The voltage accross magnets and bus bars, and the temperatures and pressures are recorded from the start of the quench until the current has decayed to zero. Thereafter the temperature and pressure recording continues at a relatively low acquisition rate.

When operating below $T_{\lambda}$ the accuracy of the cold pressure sensors is estimated to be about \pm 0.5 bar. At higher temperatures their calibration changes, sensor specific and could easily reach \pm 1 bar. The pressure sensor at D2(b) was lost. The pressure sensor reading at D2(a), and to a lesser extend D3(b), proved unreliable during a pressure calibration run at $1.9 \mathrm{~K}$. Readings from neighbouring pressure sensors were used instead.

\section{Measurements}

A dedicated series of eleven measurements was performed to permit to quantify the process of helium-mediated quench propagation. Pressure relief was set up to take place through the safety valve located at the quadrupole end of the string only. As a parameter in our measurement series we varied the set pressure value, $p_{\text {set }}$, of this pressure relief valve between 6 and 23 bar. For each set pressure we then powered Q, D1, D2, and D3 at $12.4 \mathrm{kA}$, and subsequently quenched D3 by firing its quench heaters. Figures 2 and 3 show a typical current, pressure and temperature evolution of D2 and D3 after quenching D3. After initiating a quench in D3, the current through D3 commutates to the protection diode within less than one second. The string current then decays with a time constant of $100 \mathrm{~s}$. After a delay time of several seconds up to several tens 


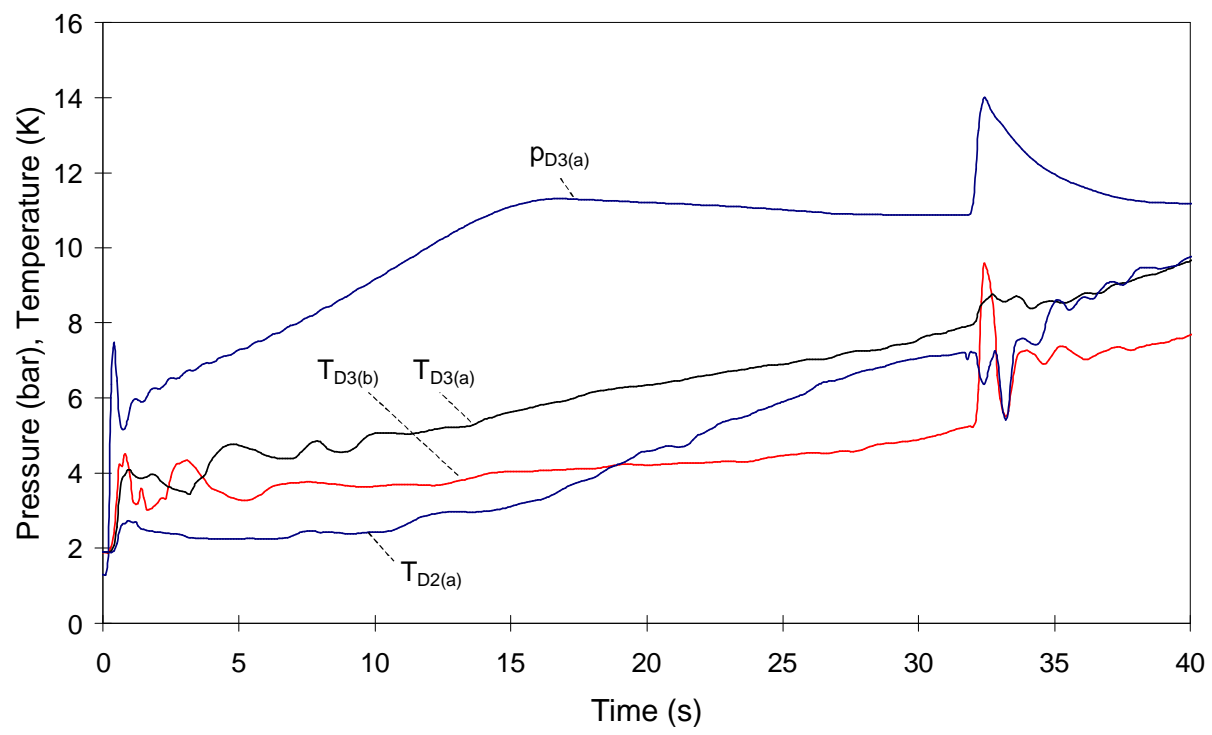

Figure 3: Quench of D3; $p_{\text {set }}=11$ bar.

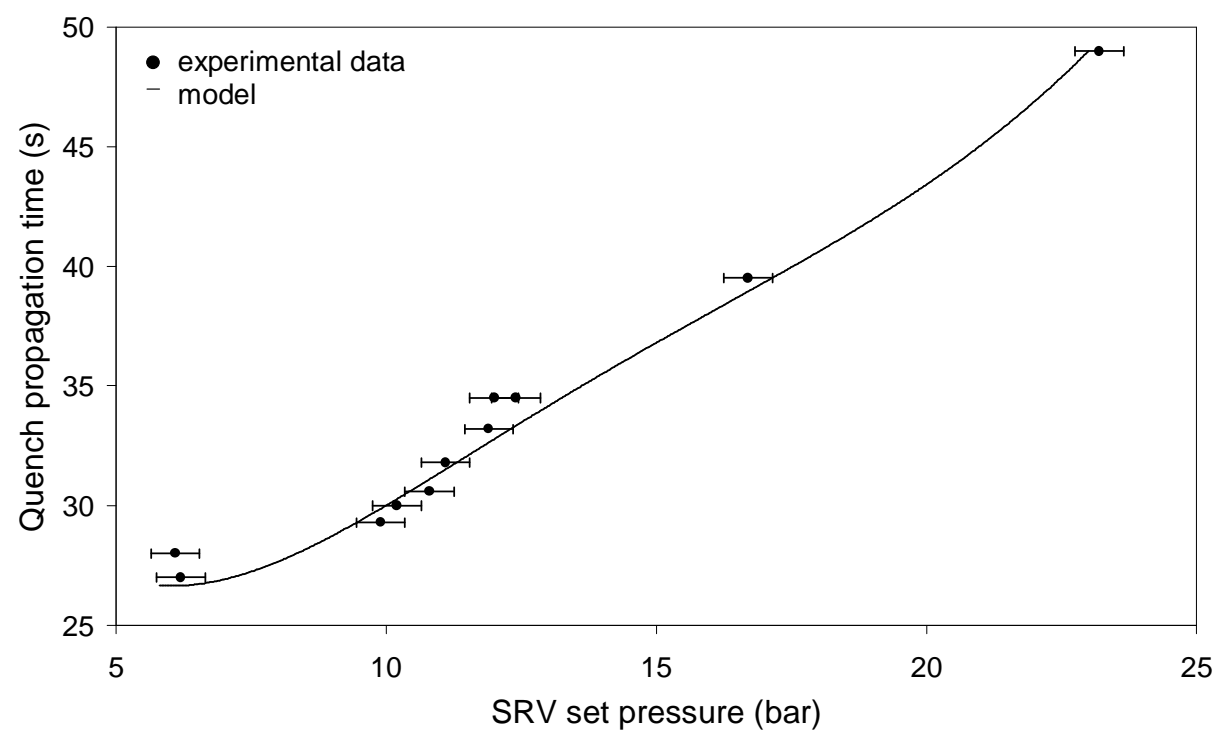

Figure 4: Data and model. 


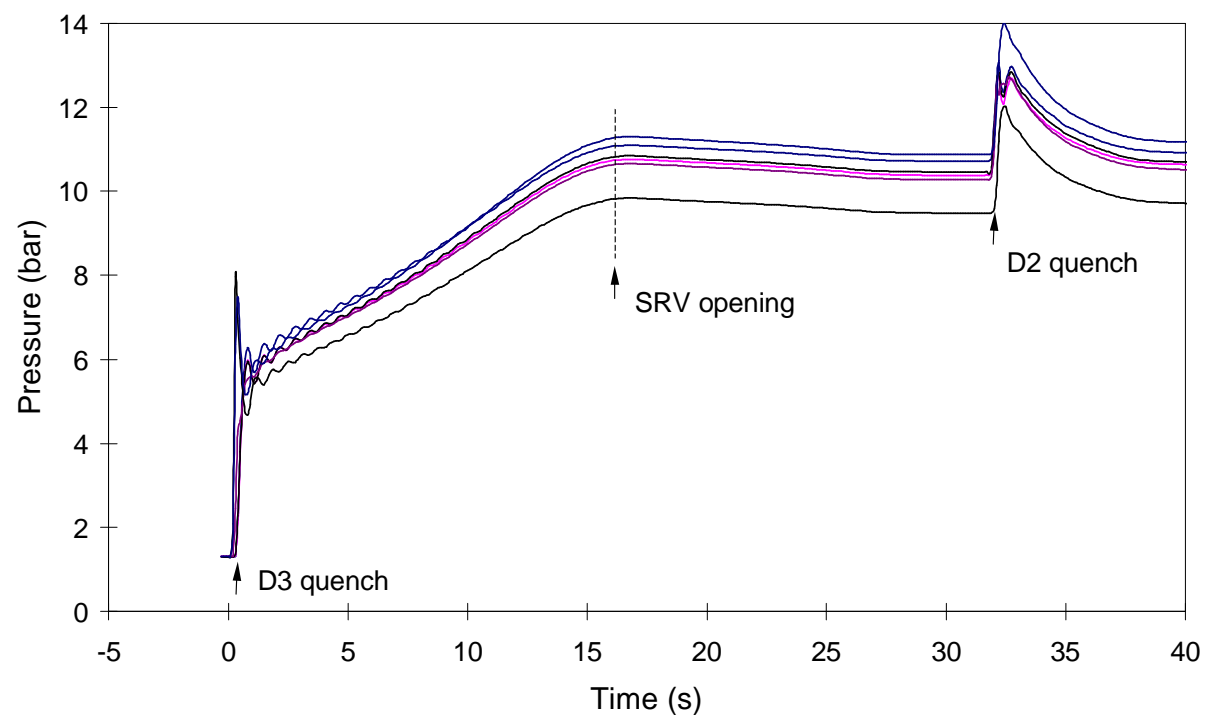

Figure 5: Pressure evolution. From above D3(a), Q(a), D1(a), Q(b), D1(b), D3(b).

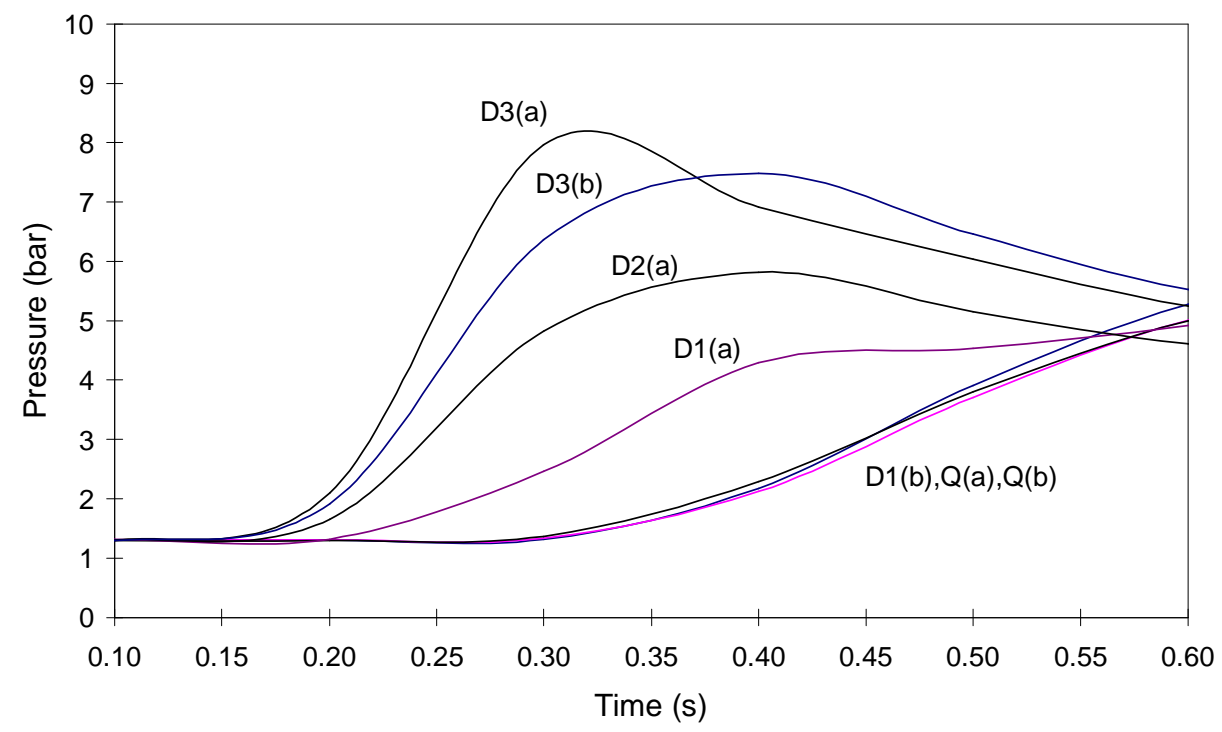

Figure 6: Pressure wave evolution. 
of seconds, depending on the pressure relief valve setting, the adjacent dipole D2 quenches. Figure 4 shows the complete series of observed quench propagation times as function of the set pressure.

As elaborated in [6] the quench process of an individual magnet can be divided into two main phases. In the first phase until about $300 \mathrm{~ms}$ after initiating the quench of D3, the temperature of its coils increases to about $100 \mathrm{~K}$. Liquid helium in close contact with the coil is heated, quickly vapourizes and expands, thereby compressing the remaining bulk helium of D3 and the rest of the string to a peak value of about 8 bar. This peak value of 8 bar is several bar lower than if D3 would have been quenched in a stand alone configuration due to the available extra helium volume in D2, D1, and Q [9]. The pressure along the string follows closely the pressure evolution in D3, delayed only by the travel time required by the pressure wave (see figure 6). Rapid evaporation and change of phase of the expanded helium cause a decrease in heat transfer between the coil and the helium marking the end of the adiabatic compression phase. Thereafter the expanded helium and the compressed helium in D3 mix, the whole string pressure drops from its peak value down to a few bar, and a second phase of slow pressure rise starts. The temperatures at both ends of D3 start to increase, followed by an even slower temperature rise in D2 at the D2-D3 interconnect side. The temperatures in the rest of the string remain unaffected. After about $16 \mathrm{~s}$ the pressure relief valve opens and maintains the pressure at $11 \mathrm{bar}$. The rate of temperature rise in D2 increases. After a further $16 \mathrm{~s}$ D2 quenches.

From figure 3 we see that temperature D2(a) rises steadily towards the value given by temperature D3(a) of the quenched magnet. This behaviour is consistent with quench propagation by the helium. In contrast to this we observe that temperature sensor D3(b), located in between D3(a) and D2(a), indicates at times beyond $18 \mathrm{~s}$ values much below the other two. We attribute this to calibration data errors and will ignore this sensor for the remainder of the analysis.

\section{Process identification}

Heat conduction via the bus bars is excluded as the responsible propagation mechanism because with bus bar lengths of about $10 \mathrm{~m}$ in between D3 and D2 the resultant delay time would have been much longer than those actually measured [2]. This is substantiated by the voltage taps measurements that indicate that the resistive transition of D2 initiates at its coils ends at the D2D3 connection side whereas the bus bars connection to the D2 coils is situated at the D2-D1 interconnect side [10]. We conclude that mediation by the helium is the process responsible for the quench propagation to D2.

Figure 7 shows in a phase diagram the measured evolution of the helium state in D3, in D2(a), and in the rest of the string. The lines corresponding to ideal isochoric heating and ideal adiabatic compression are drawn for reference. As described in the previous paragraph we see that D3 initially evolves near the adiabatical compression line, it then very soon starts to deviate and joins the isochoric heating line. The rest of the string behaves as being adiabatically compressed. Only the portion of the string near the D2-D3 interconnect (D2(a)) starts to deviate at a certain moment from the pure adiabatic compression line, signalling the arrival of warm helium. 


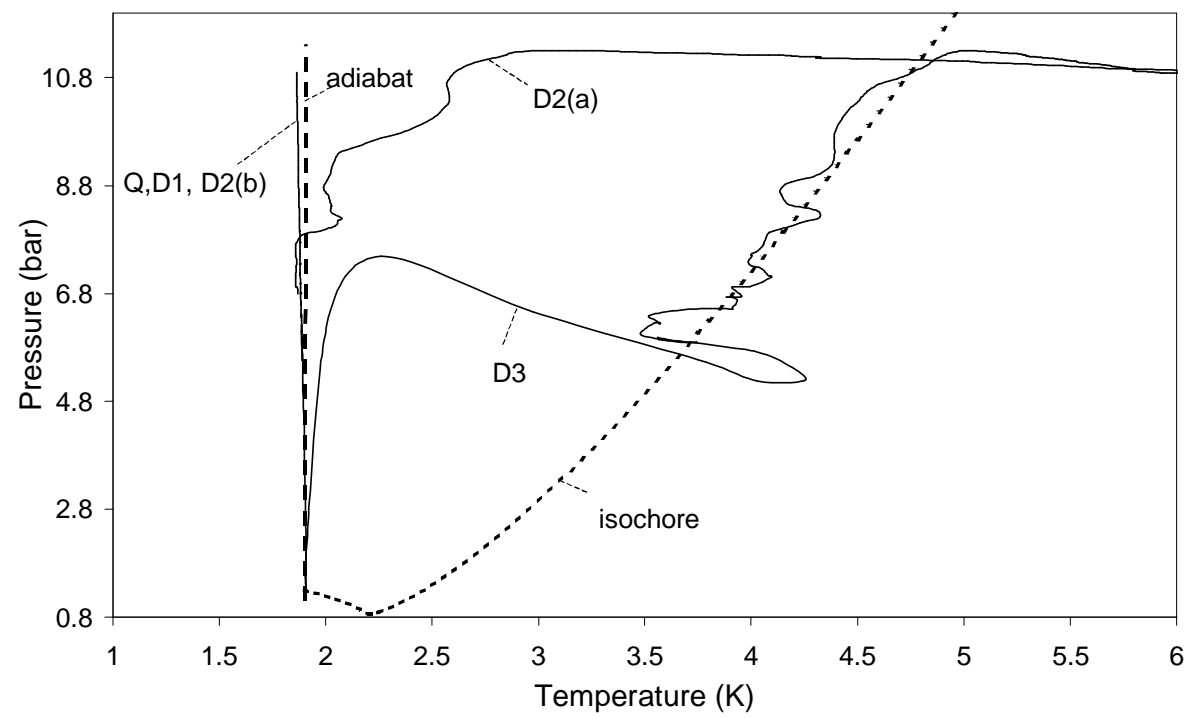

Figure 7: Phase diagram of the helium evolution relative to adiabatic compression and isochoric heating.

\section{Process modeling}

\section{Initial state}

Since until the the start of the second phase of the quench process the temperature in D2 is hardly affected, we start our model description from this phase. We take as initial conditions the state at $1.5 \mathrm{~s}$ after the quench when the effects of the adiabatic compression phase of D3 have died out. The string is subdivided into two parts. The first part representing the quenched dipole, D3, and the second part the rest of the string:

$$
\begin{array}{ll}
t_{0} & =1.5 \mathrm{~s} \\
p\left(t_{0}\right) & =5.8 \mathrm{bar} \\
T_{\mathrm{D} 3}\left(t_{0}\right) & =3.5 \mathrm{~K} \\
T_{\mathrm{rst}}\left(t_{0}\right) & =1.9 \mathrm{~K}
\end{array}
$$

where the subscript rst refers to parameters concerning the rest of the string.

\section{Power deposition}

Figure 8 shows the power deposited in the helium of D3 when there is no pressure relief. It is determined from the change in internal energy with time given by the temperature and pressure evolution measured at the respective locations $a$ and $b$ of D3. The ripples in the pressure evolution, visible between $t_{0}$ and about $12 \mathrm{~s}$ of figure 5 , represent local deviations from the general trend due to pressure waves. The magnitude of these ripples is such that applying these local pressure values to deduce the power deposited in the total amount of helium in D3 would yield unphysically large power fluctuations. To obtain, for times between $t_{0}$ and about $12 \mathrm{~s}$, values 


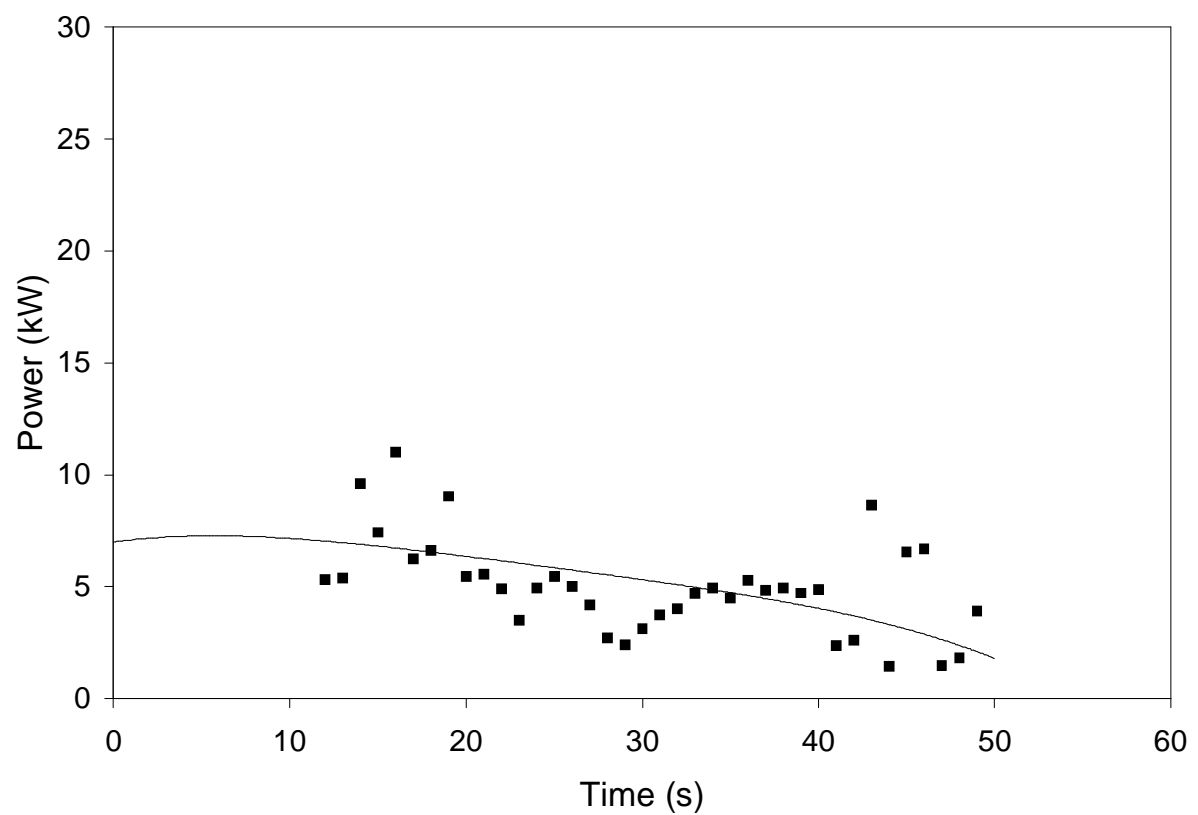

Figure 8: Power deposition in D3, unfiltered and filtered data.

representative for the power deposition in D3 as a whole we therefore used the pressure values given by the trend-line of the pressure evolution instead. This is plotted using a straight line in figure 8 .

When there is no pressure relief, the power into the rest of the string is mainly due to compression work driven by the expansion of the helium contained in D3. It is about $0.7 \mathrm{~kW}$ average. When pressure relief takes place the enthalpy flow out of the system compensates for this compression energy.

\section{Model}

Figure 9 shows the typical lenghts and cross-sectional areas involved. $\left\{A_{\mathrm{i}}, L_{\mathrm{i}}\right\},\left\{A_{\mathrm{e}}(a), L_{\mathrm{e}}(a)\right\}$, $\left\{A_{\mathrm{e}}(b), L_{\mathrm{e}}(b)\right\}$, and $\left\{A_{\mathrm{y}}, L_{\mathrm{y}}\right\}$ are the cross-sections occupied by the helium at the interconnect, the magnet cold mass end cap, and the magnet yoke repectively and their corresponding lengths. The geometric information contained in $A_{\mathrm{i}}, A_{\mathrm{e}}(a), A_{\mathrm{e}}(b)$, was obtained from construction drawings, whereas $A_{\mathrm{y}}$ was determined from volume measurement of the dipole coldmass. The distance from the yoke end plate until the nearest coil peak field location, $L_{\mathrm{c}}$, where the coil bending goes over into the straight part, is about 140-150 mm [11].

Pressure waves are not essential to the quench propagation process. For the purpose of our model we therefore consider the pressure to be homogeneous over the full length of the string. This means that we neglect effects below a time scale of the order $L_{\text {rst }} / v_{\text {sound }} \approx 150 \mathrm{~ms}$. The helium content in the buffer volume formed by the space between the interconnect and the quench location is estimated to be $0.0143 \pm 0.001 \mathrm{~m}^{3}$. This is about $2 \%$ of the volume of the rest of the string. With the exception of this buffer volume the temperatures in the rest of the string are 


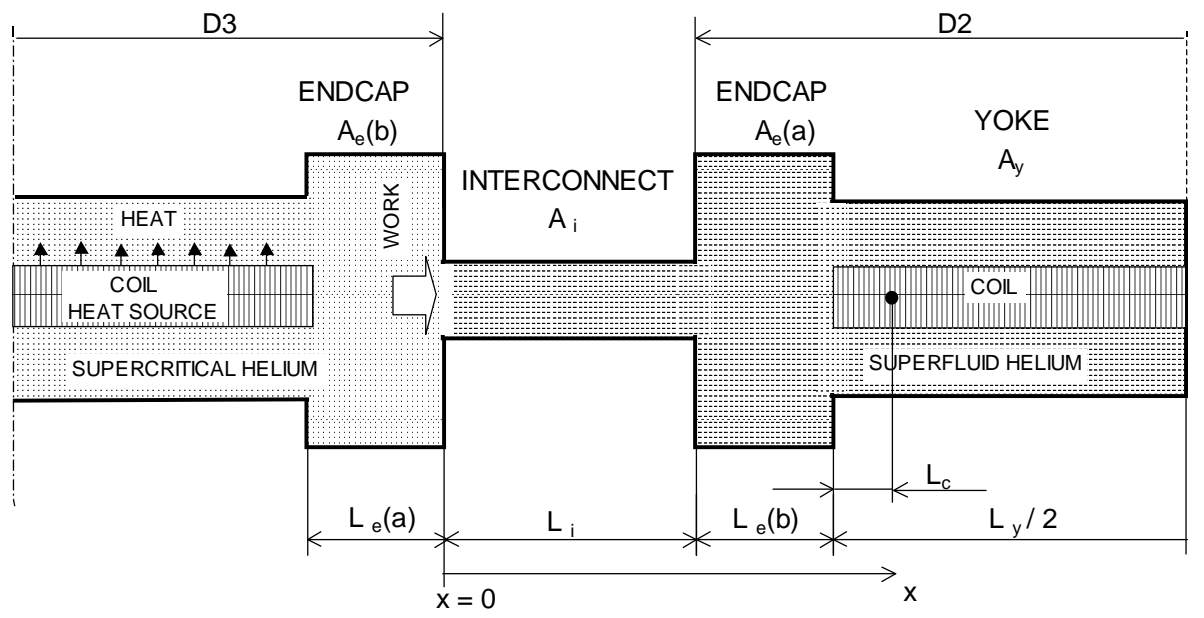

Figure 9: Model schematic with interactions shown.

homogeneous (see figure 2).

The foregoing considerations made us choose a two stepped aproach. In the first step we calculate the pressure and bulk temperature evolution of D3 and of the rest of the string by a two-node model. In the second step we use the resultant pressure and temperature evolution of D3 as input to a one-dimensional flow model to calculate the mass and enthalpy flow out from D3 over the interconnect and into D2.

Two-node model: To calculate the pressure evolution over a small time step we assume that the heat $d Q$ deposited in D3 during this time is absorbed by the helium in D3 up to the boundary between the D3 endcap and the D3-D2 interconnect. This helium expands, and by doing so adiabatically compresses the helium contained in the rest of the string. The helium equation of state, the energy balance equations together with the condition of pressure homogeneization and the assumption of adiabatic compression are sufficient to calculate the new pressures and temperatures in D3 and the rest of the string (equations 1 to 4):

$$
\begin{aligned}
\frac{\partial U_{\mathrm{D} 3}}{\partial t} & =\frac{\partial Q}{\partial t}-p_{\mathrm{D} 3} \frac{\partial V_{\mathrm{D} 3}}{\partial t} \\
\frac{\partial U_{\mathrm{rst}}}{\partial t} & =-p_{\mathrm{rst}} \frac{\partial V_{\mathrm{rst}}}{\partial t} \\
p_{\mathrm{D} 3} & =p_{\mathrm{rst}} \\
d V_{\mathrm{D} 3} & =-d V_{\mathrm{rst}} .
\end{aligned}
$$

A fraction of helium of D3 is expelled into the interconnect. At the next time step it is then no longer directly heated by the power from the quenched magnet. From the moment the safety relief valve opens, it effectively fixes the string pressure at the value $p_{\mathrm{D} 3}=p_{\text {rst }}=p_{\text {set }}$ determined by the pressure setting of the valve. The evolution of D3 changes from isochoric to isobaric, which is taken into account by changing equation 2 into equation 5 :

$$
\frac{\partial U_{\mathrm{rst}}}{\partial t}=-p_{\mathrm{rst}} \frac{\partial V_{\mathrm{rst}}}{\partial t}+h_{\mathrm{rst}} \frac{\partial m_{\mathrm{rst}}}{\partial t} .
$$




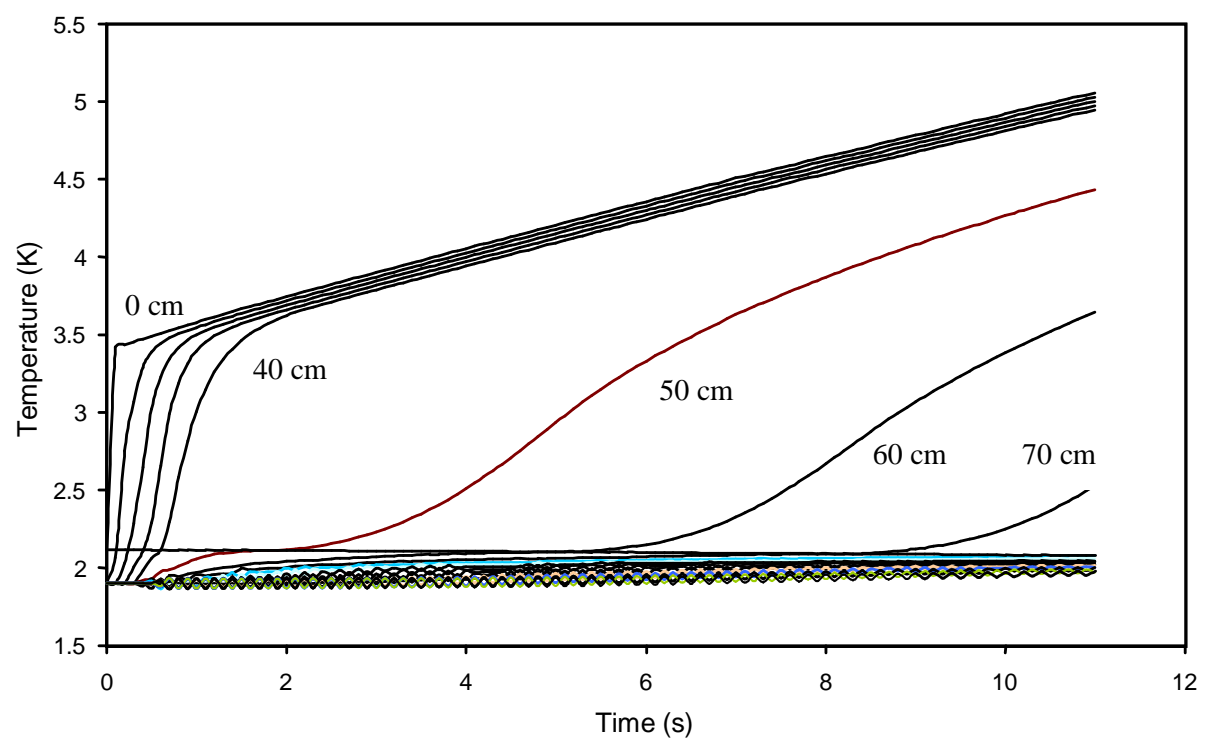

Figure 10: Evolution of temperature profile; $p_{\text {set }}=17$ bar.

As a consequence the mass flow out of D3 increases with respect to the isochoric evolution.

One-dimensional flow model: The gradual expulsion of increasingly warmer helium from D3 into the interconnect is calculated from the equations of continuity for mass and enthalpy, with the pressure $p$ and the boundary values for $\rho$ and $h$ at $x=0$ given by the results of the two-node model (equations 6 to 10):

$$
\begin{aligned}
\frac{\partial \rho}{\partial t} & =-\frac{\partial \rho v}{\partial x}, \\
\frac{\partial \rho h}{\partial t} & =-\frac{\partial \rho v h}{\partial x}, \\
p(x, t) & =p_{\mathrm{D} 3}(t), \\
\rho(0, t) & =\rho_{\mathrm{D} 3}(t), \\
h(0, t) & =h_{\mathrm{D} 3}(t) .
\end{aligned}
$$

After solving for $h(x, t)$ and $\rho(x, t)$ the temperature profile $T(x, t)$ is determined from $h(x, t)$ and $p(t)$ via the helium equation of state. Figure 10 shows an example of the calculated evolution of temperature profile up to $70 \mathrm{~cm}$ from the D3 interconnect. As the warm front reaches the location of the coil in D2(a) which exhibits the peak field before the current has decayed to zero, D2 will quench once the temperature gets above the coil critical temperature.

In our model the temperature profile from D3 to D2 is the result of gradual expulsion of increasingly warmer helium from D3 into the interconnect. Initially we did consider the contribution of pure heat conduction due to the temperature gradient in the helium to the warming up of D2. However with the given dimensions and temperature gradients, conduction is completely negligable. 


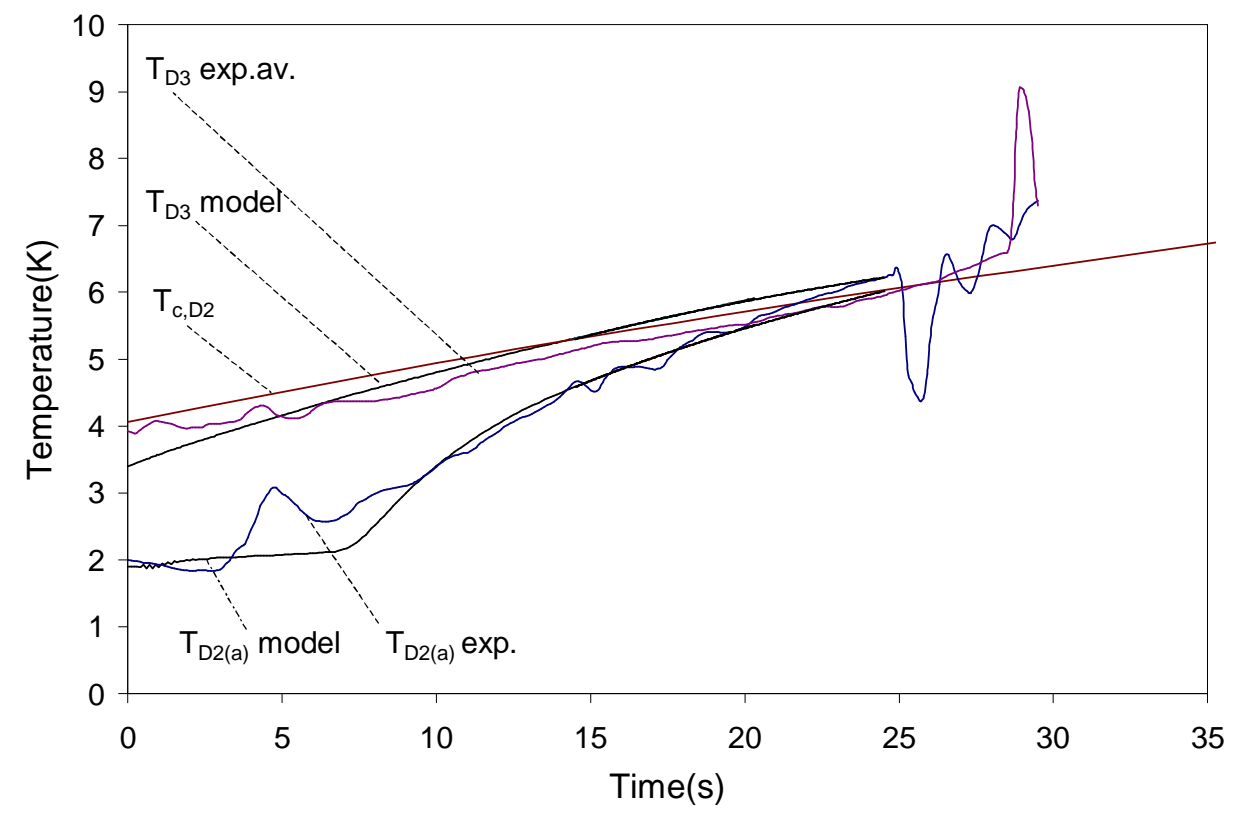

Figure 11: Model and data for quench at $12.4 \mathrm{kA} ; p_{\text {set }}=6$ bar.

\section{Comparison with experiments}

Figure 4 shows the measured quench propagation time together with the calculated time according to our model. Figure 11 compares the model to a quench propagation measurement with the $\mathrm{SRV}$ relief pressure set at 6 bar. $T_{\mathrm{c}, \mathrm{D} 2}$ indicates the critical temperature of D2 as function of time when ramping down the current [2]. The correspondence between the model-predicted quench propagation time and the measured one is remarkebly good. The measured scatter could, apart from the uncertainty in the pressure measurements, have its origin in slight changes of quench location, since this would directly correspond to a change in helium volume to be heated up.

\section{Effect of variation of power and of helium content}

The string contains specific LHC prototype dipole magnets of cold mass length $10.9 \mathrm{~m}$, and of helium volume $0.228 \mathrm{~m}^{3}$. We characterise a single dipole quench in this geometry by the ratio $R_{\mathrm{q}}=V_{\text {dipole }} / V_{\text {string }}$ of the quenched volume to total volume ratio.

The curves in figure 12 labelled $R_{\mathrm{q}}=0.27$ show the calculated dependance of the quench propagation time in the string on varying power deposition per unit volume into D3, and on a change in helium content in the buffer volume formed by the space between the interconnect and the quench location at a pressure relief setting of 17 bar. The variations are indicated as ratios with respect to the string parameters. At low power deposition the quench propagation time will become infinite. 


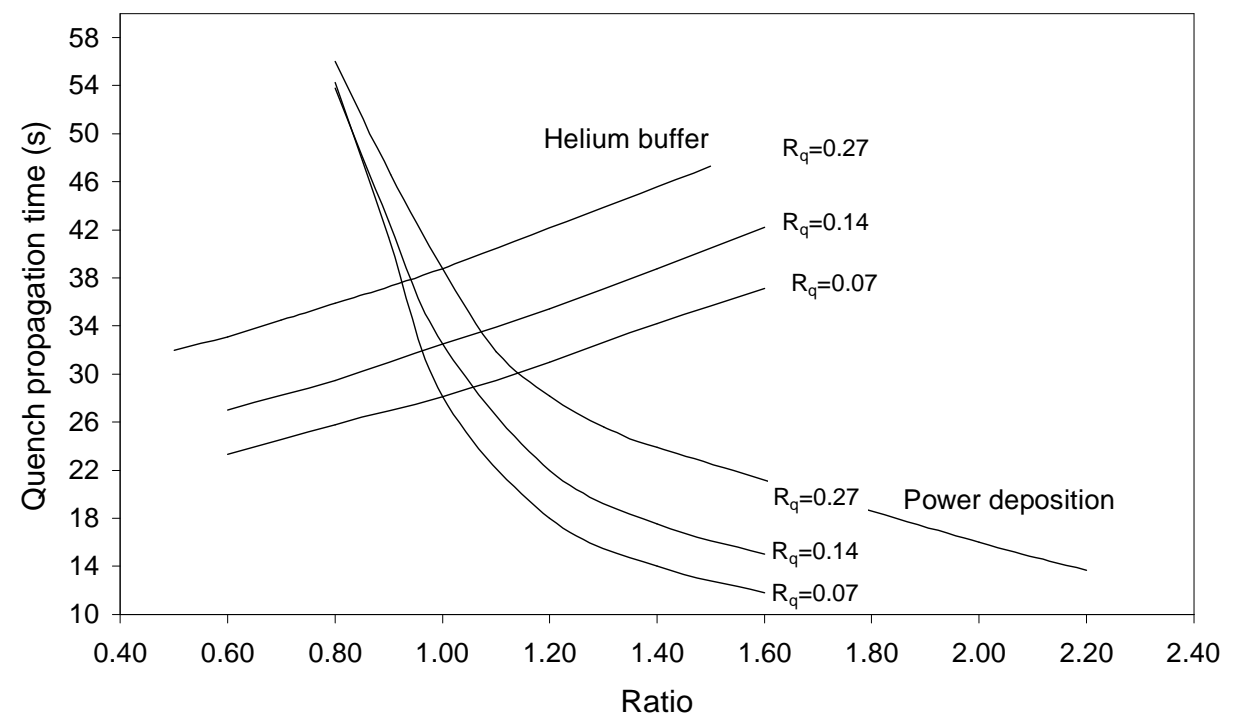

Figure 12: Propagation time as function buffer volume and power deposition; $p_{\text {set }}=17$ bar.

\section{Scaling to other string geometries}

We applied the model to predict the behaviour of strings differing in the quenched volume to total volume ratio $R_{\mathrm{q}}$, but assembled out of similar magnets. Under similar magnets we understand magnets having identical power deposition per unit volume as the LHC prototype magnet D3, and with the same buffer volume. This correspondence is defined at ratio=1 for each of the $R_{\mathrm{q}}$ labelled curves in figure 12. $R_{\mathrm{q}}=0.14$ is the estimated ratio for a $107 \mathrm{~m}$-long LHC cell in which a $15 \mathrm{~m}$ long dipole magnet quenches. $R_{\mathrm{q}}=0.07$ is the estimated ratio for a $214 \mathrm{~m}$-long double LHC cell in which a $15 \mathrm{~m}$ long dipole magnet quenches. The latter case corresponds to the longest hydraulic unit in the present LHC machine design.

The calculated quench propagation time decreases with decreasing $R_{\mathrm{q}}$. The reason is that because of the relative increase in volume of the rest of the string compared to the quenched dipole volume and the power deposition therein, at a given pressure it becomes more compressed than before. The gradual expulsion of warm helium from the quenched magnet into the rest of the string is facilitated, and hence a reduced quench propagation time.

\section{Conclusions and recommendations}

We have shown that quench propagation in the string is adequatly explained by the gradual expulsion of warm helium from the quenched magnet into the neighbouring one driven by the power deposited into the helium of the quenched magnet. As long as there is no pressure relief yet, this expulsion works against the confined helium of the rest of the string, thereby compressing it adiabatically. This is a favourable situation, because as soon as there is pressure relief the helium expulsion takes place isobarically, which is significantly faster. This dependence on pressure relief is roughly $1.4 \mathrm{~s} / \mathrm{bar}$. Therefore for the LHC this pressure relief should preferably be put at 
the highest possible value. The model showed the strong buffer effect of the combined helium content in the end volumes of the magnet cold masses and the volume inside the yoke up to the coil location with lowest temperature margin. Purposely increasing this volume could be envisaged if quench propagation is a problem in a certain magnet configuration. The dependence on power deposition is about as strong. However since this power deposition is intimately linked to the magnet construction details it will be much harder to influence this.

\section{Acknowledgements}

We acknowledge the work of the string-team members of the LHC prototype test string-1, and in particular of L. Coull, F. Rodriguez-Mateos and R. Schmidt, in performing the quench propagation measurements upon which we could base our modelling.

\section{References}

[1] L. Coull, D. Hagedorn, G. Krainz, F. Rodriguez-Mateos, R. Schmidt, Quench propagation tests on the LHC superconducting magnet string, $5^{\text {th }}$ European Particle Accelerator Conference - EPAC '96, Sitges, Barcelona, Spain, 10-14 June 1996, / Ed. by S. Myers, A. Pacheco, R. Pascual, C. Petit-Jean-Genaz, and J. Poole, - IOP, Bristol, 1996.

[2] F. Rodriguez-Mateos, R. Schmidt, L. Serio, Thermo-hydraulic quench propagation at the LHC superconducting magnet string, $17^{\text {th }}$ International Cryogenic Engineering Conference (ICEC-17), Bournemouth, UK, 14-17 July 1998 /Ed. by D. Dew-Hughes, R. G. Scurlock, and J. H. P. Watson, - IOP, Bristol, 1998.

[3] M. Chorowski, W. Erdt, Ph. Lebrun, G. Riddone, L. Serio, L. Tavian, U. Wagner and R. van Weelderen, A simplified cryogenic scheme for the Large Hadron Collider, Cryogenic Engineering Conference 1997, Portland, Adv. Cryog. Eng., A : 43 (1998).

[4] B. Szeless, F. Rodriguez-Mateos, F. Calvone, Development of industrially produced composite quench heaters for the LHC superconducting lattice magnets, $16^{\text {th }}$ International Cryogenic Engineering Conference / International Cryogenic Materials Conference, Kitakyushu, Japan, 20-24 May 1996 / Ed. by T. Haruyama, T. Mitsui, and K. Yamafuji, Elsevier, Amsterdam, 1997.

[5] L. Coull, R. Denz, D. Hagedorn, High current diffusion type diodes at cryogenic temperatures for the LHC superconducting magnet protection, Adv. Cryog. Eng. A : 43 (1998).

[6] M. Chorowski, Ph. Lebrun, L. Serio and R. van Weelderen, Thermohydraulics of quenches and helium recovery in the LHC prototype magnets strings, Cryogenics Vol. 38 no. 5 (1998)

[7] A. Bézaguet, J. Casas Cubillos, H Guinaudeau, B. Hilbert, Ph. Lebrun, L. Serio, A. Suraci, and R. van Weelderen, Cryogenic operation and testing of the extended LHC prototype 
magnet string, $16^{\text {th }}$ International Cryogenic Enegineering Conference / International Cryogenic Materials Conference, Japan, 20-24 May 1996 / Ed. by T. Haruyama, T. Mitsui and K. Yamafuji, K-Elsevier, Amsterdam, 1997.

[8] P. Bonnal et al., The String Shutdown Report LHC-Project-Note-107, 1997, CERN.

[9] G. Gerin, B. Vullierme and R. van Weelderen, Measurement of the Thermohydraulic Behaviour of LHC Dipole Prototypes after a Quench, Advances in Cryogenic Engineering Vol. 41 (1996), pp. 811-818.

[10] F. Rodriguez-Mateos, CERN private communication, August 2000.

[11] A. Siemko, CERN private communication, September 2000. 